\title{
Rigidity of Spreadings and Fields of Definition
}

\author{
Chris Peters \\ Technical University Eindhoven \\ and Université Grenoble Alpes \\ email: c.a.m.peters@tue.nl
}

November 2016

\begin{abstract}
Varieties without deformations are defined over a number field. Several old and new examples of this phenomenon are discussed such as Belyı̆ curves and Shimura varieties. Rigidity is related to maximal Higgs fields which come from variations of Hodge structure. Basic properties for these due to P. Griffiths, W. Schmid, C. Simpson and, on the arithmetic side, to Y. André and I. Satake all play a role. This note tries to give a largely self-contained exposition of these manifold ideas and techniques, presenting, where possible, short new proofs for key results.

AMS Classification $11 \mathrm{G}_{18},{ }_{14} \mathrm{C}_{30},{ }_{14} \mathrm{Do} 7,{ }_{14} \mathrm{G}_{35}$
\end{abstract}

\section{Introduction}

Results stating that certain types of algebraic varieties are definable over a number field are scattered in the literature. Arguably, those most studied form the class of Shimura varieties [Sh, F, Mi]. Another famous example is Belyî's theorem [Bel] which characterizes curves over $\overline{\mathbf{Q}}$ as those which have a Belyı representation, i.e., a branched cover of the line branched in exactly three points. In dimension two, the fake projective planes [ $\mathrm{Pr}-\mathrm{Y}$, 
Pr-Y-2] and the Beauville surfaces [Be, Exercise X.13 (4)], [Ba-C-G] are known to have models over $\overline{\mathbf{Q}}$.

Such examples can be uniformly explained by constructing a suitable spread of the varieties concerned as demonstrated in Sections 1.3 and 2 ,

Of a totally different flavor are the applications to special subvarieties of Shimura varieties in Section 3.5 on the one hand, and to splittings of Higgs bundles as given in Section 4.3 on the other hand. As has been known since the work of Viehweg and Zuo [Vie-Z], the last two are just facets of the same phenomenon: Higgs bundles of a very special kind, those that they called "maximal" are directly related to special subvarieties of certain Shimura varieties. One of the goals of this note is to show that rigidity plays a central role in this; exploiting this, simplifies several of the arguments.

The overall goal of this note is to show how a few relatively simple ideas plus some standard techniques from deformation theory and Hodge theory explain a wide range of phenomena of the above kind. It brings together various known results from very different subfields of mathematics. This is the reason why I thought to explain some of the basic notions and techniques from these fields, and also to search for new simpler proofs.

This note has been inspired by discussions with Stefan Müller-Stach. Equally influentual has been [G-G, Ch. 4] as well as the last chapter of [Mu-O]. Finally I would like to thank Christopher Deninger for pointing out the references [E, Sh]. 


\section{Contents}

1 Spreads of varieties and rigidity 3

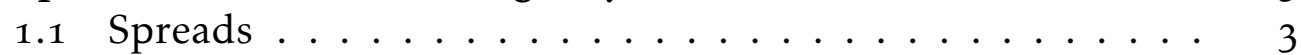

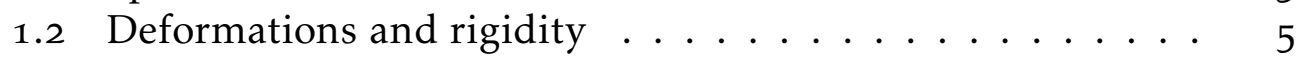

1.3 Rigidity and fields of definition ........... 8

2 Further examples of models over number fields 11

2.1 Locally symmetric spaces . . . . . . . . . . . . . . 11

2.2 Holomorphic maps into locally symmetric spaces . . . . 12

3 Applications to variations of Hodge structure 13

3.1 Hodge theory revisited ............... 13

3.2 Application to variations of weight 1 and $2 \ldots \ldots 15$

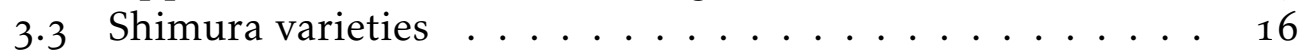

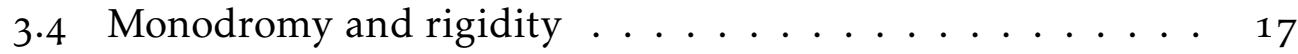

3.5 Special subvarieties of Shimura varieties ........ 21

4 Applications to Higgs bundles 23

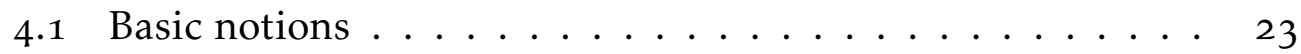

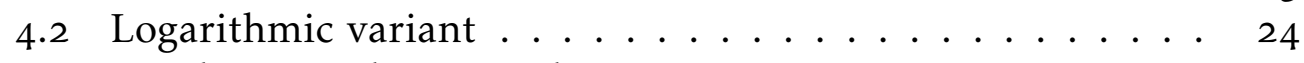

4.3 Rigid maximal Higgs subsytems ............ 25

\section{Spreads of varieties and rigidity}

\subsection{Spreads}

The "spread philosophy" roughly states that a complex algebraic variety can be seen as a family over a base variety determined by specifying some transcendence basis of the field of definition of the variety. Spreads are by no means unique but all share the crucial property that, by construction, the total family is always defined over a number field.

Although this construction can be phrased in varying generality [G-G, $\S 4.1]$, the following somewhat restricted version suffices for this note.

Proposition 1.1. Let $X$ be smooth complex quasi projective variety. There exists a smooth family $f: X \rightarrow S$ defined over $\overline{\mathbf{Q}}$ such that

1. $X, S$ are smooth quasi-projective; 
2. there is a canonical point $o \in S$ such that $f^{-1} o=X$;

3. if $s \in S$ is a $\overline{\mathbf{Q}}$-rational point, the fiber $X_{s}=f^{-1} s$ is defined over $\overline{\mathbf{Q}}$.

Proof. Suppose for simplicity that the variety $X$ is projective and is given by a finite set of polynomials. The coefficients of these polynomials generate a field $k$ of finite transcendence degree $r$ over $\mathbf{Q}$, say $k=k_{0}\left(\alpha_{1}, \ldots, \alpha_{r}\right)$ where $\left\{\alpha_{1}, \ldots, \alpha_{r}\right\}$ is a transcendence basis for $k$ and where $k_{0}$ is a number field, say of the form $\mathbf{Q}\left[x_{0}\right] / P$ with $P$ some monic irreducible polynomial. Then $k$ is the function field of some complex algebraic variety $S^{\prime}$. The deformation will be constructed over a Zariski open subset $S$ of $S^{\prime}$.

The basic idea is to replace the coefficients $\alpha_{j}$ of each of the polynomials defining the variety $X$, by variables $x_{j}$. A point $s \in S^{\prime}$ corresponds to a field $k(s)$ isomorphic to $k$. If one replaces the coefficients in $k$ of a defining set of homogeneous equations for $X$ by the corresponding coefficients in $k(s)$ one gets a variety $X_{s}$. The $X_{s}$ glue to a variety $\mathscr{X}$ fibered over $V(P)$. Indeed, it is given by the same equations as $X$ except that the coefficients for these equations are not considered as numbers but as $\mathbf{Q}$-polynomials in the supplementary variables $x_{j}$ tied by the extra equation $P\left(x_{0}, \ldots, x_{r}\right)=0$.

Substituting $x_{j}=\alpha_{j}$ gives a canonical $k$-valued point $o \in S^{\prime}$ and by construction $X_{o}=X$. Since $k / k_{0}$ is separable, this point is a non-singular point. Now replace $S^{\prime}$ by a suitable Zariski open neighborhood $S$ of $o$ such that the variety $S$ is smooth. Again by separability, this variety is smooth along $X_{o}$. But it might still be singular or reducible. To remedy this, first take the component of $X$ which contains $f^{-1} o$. Then replace $S$ by a smaller neighborhood of $o$ such that not only the total space is smooth, but also all of the fibers of the fibration are smooth. The resulting family, still denoted $f: X \rightarrow S$, is a smooth deformation of $X$. By construction, the Zariski-open subsets figuring in the construction are complements of equations over $\overline{\mathbf{Q}}$, and so the resulting family is defined over $\overline{\mathbf{Q}}$.

Finally, since $\overline{\mathbf{Q}}$ is algebraically closed, $S$ contains points $s$ defined over $\overline{\mathbf{Q}}$. This amounts to replacing the variables $x_{j}$ figuring in the coefficients for the equations of $X$ by suitable algebraic numbers and hence $X_{s}$ is defined over $\overline{\mathbf{Q}}$.

Remark 1.2. There are several variants of this result: one can spread pairs $(X, Z)$ with $Z$ a closed subvariety of $X$. Similarly, one can spread a given morphism $f: X \rightarrow Y$ between varieties. 


\subsection{Deformations and rigidity}

Let me first recall some basic definitions and facts. More details and proofs can be found for example in [Sern].

\section{Kodaira-Spencer classes}

A complex variety $X$ is said to be infinitesimally, respectively locally rigid if any infinitesimal deformation of $X$, resp. any local deformation $p: X \rightarrow$ $S$ of $X$ with $S$ sufficiently small, is trivial, i.e. isomorphic to the product deformation. This can be rephrased by saying that if, say $o \in S$ is such that

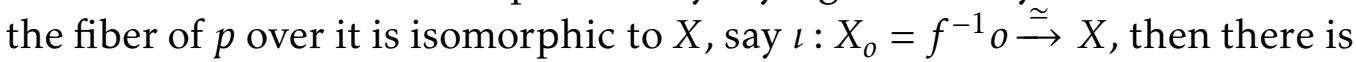
a morphism $S \rightarrow \operatorname{Aut}(X), \quad s \mapsto g_{s}, g_{o}=\mathrm{id}_{X}$ inducing a product structure on the family $X \rightarrow S$ :

$$
X \times S \stackrel{\simeq}{\longrightarrow} X, \quad(x, s) \mapsto\left(\log _{s}(x), s\right) .
$$

As is well known, a variety $X$ is indeed infinitesimally or locally rigid if $H^{1}\left(X, \Theta_{X}\right)=0$. If such a variety appears in a deformation $p: X \rightarrow S$ of $X \simeq X_{o}, o \in S$, finer information is present by looking at the the KodairaSpencer map. Recall that it is given as the extension class of the exact sequence

$$
0 \rightarrow T_{o}(S) \otimes \Theta_{X} \rightarrow \Theta_{X} \mid X \rightarrow \Theta_{X} \rightarrow 0
$$

of $\sigma_{X}-$ modules. In other words, it gives a characteristic map

$$
\kappa_{p}: T_{o}(S) \rightarrow H^{1}\left(X, \Theta_{X}\right) .
$$

For a given deformation, it measures deviation of triviality of the deformation:

Theorem 1.3 ([K-S, Thm. 18.3]). Suppose that a family $p: X \rightarrow S$ is regular in the sense that $\operatorname{dim} H^{1}\left(X_{s}, \Theta_{X_{s}}\right)$ is constant for $s \in S$. Then it is trivial if and only if $\kappa_{p}=0$.

Observe that this theorem gives back the criterion that $X$ is rigid if and only if $H^{1}\left(X, \Theta_{X}\right)=0$. Indeed, if this is the case, by semi-continuity, any sufficently small deformation of $X$ is regular and the theorem applies to show rigidity. 


\section{Variants}

1. Infinitesimal deformations of pairs $(X, Z)$ with $Z$ a closed subscheme of a smooth variety $X$. Any such deformation $p$ with base $(S, o)$ (i.e. with fiber over $o$ isomorphic to $(X, Z))$ is classified by its Kodaira-Spencer map

$$
\kappa_{p}: T_{o} S \rightarrow H^{1}\left(X, \Theta_{X}(Z)\right),
$$

where $\Theta_{X}(Z)$ is the sheaf of germs of vector fields on $X$ tangent to $Z^{1}$. This deformation is rigid precisely when $\kappa_{p}=0$ as before.

2. Deformations of morphisms $f: X \rightarrow Y$. These are given by a commutative diagram

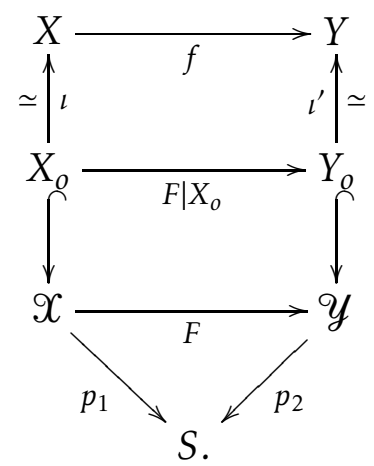

A deformation of morphism as above is a deformation keeping the source, respectively target fixed if $p_{1}$ resp. $p_{2}$ are a trivial deformations. A morphism $f$ is rigid, if all infinitesimal deformations of $f$ are trivial in the sense that there are morphisms

$$
\begin{aligned}
& S \rightarrow \operatorname{Aut}(X) \quad s \mapsto g_{s}, g_{o}=\operatorname{id}_{X} \\
& S \rightarrow \operatorname{Aut}(Y) \quad s \mapsto g_{s}^{\prime}, g_{o}^{\prime}=\operatorname{id}_{Y} .
\end{aligned}
$$

which trivialize the deformation: for all $s \in S$ there is a commutative diagram

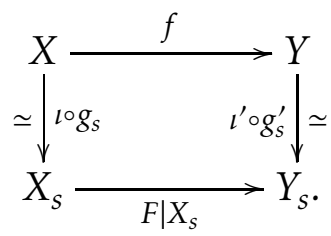

Two special cases will be used in this note:

${ }^{1}$ This is Sernesi's notation; if $Z$ is a normal crossing divisor it is dual to $\Omega_{X}^{1}(\log Z)$ and other auhors use $\Theta_{X}(-\log Z)$ in this case. 
a) Deformations of a morphism $f: X \rightarrow Y$ between non-singular varieties keeping source and target fixed. Such morphisms are classified by the vector space $H^{0}\left(X, f^{*} \Theta_{Y}\right)$.

b) Deformations of closed embeddings $f: Z \hookrightarrow X$ between smooth varieties with target fixed. Here the characteristic morphism is

$$
\kappa_{F}: T_{o} S \rightarrow H^{0}\left(Z, N_{Z \mid X}\right),
$$

where $N_{Z \mid X}$ is the normal bundle of $Z$ in the ambient manifold $X$. Note that automorphisms of $X$ yield non-trivial deformations of $f$ but these are trivial as deformations of $Z$ itself. Indeed, there is an exact sequence

$$
0 \rightarrow H^{0}\left(Z, \Theta_{Z}\right) \stackrel{i^{*}}{\rightarrow} H^{0}\left(Z, \Theta_{X} \mid Z\right) \rightarrow H^{0}\left(Z, N_{Z \mid X}\right) \stackrel{\delta}{\rightarrow} H^{1}\left(Z, \Theta_{Z}\right)
$$

The quotient $H^{0}\left(\Theta_{X} \mid Z\right) / i^{*} H^{0}\left(\Theta_{Z}\right)$ is the space of isomorphisms classes of infinitesimal deformations of $f$ keeping $Z$ and $X$ fixed; the next term in the sequence, $H^{0}\left(X, N_{Z \mid X}\right)$, is the space of infinitesimal deformations of $f$ keeping only $X$ fixed and $\delta$ maps such a deformation to the corresponding deformation of $Z$, i.e., it is the forgetful map. The embedding $f$ is rigid in this case precisely if $\kappa_{F}=0$. If $Z$ itself is rigid, this would follow if $H^{0}\left(Z, \Theta_{Z}\right) \rightarrow H^{0}\left(Z,\left.\Theta_{X}\right|_{Z}\right)$ is surjective. In case $Z$ admits no vector fields, this means $H^{0}\left(Z,\left.\Theta_{X}\right|_{Z}\right)=0$. For later use, consider the following special case, that of a totally geodesic submanifold $Z$ of $X$ :

Proposition 1.4 ([Ca-MS-P, Sect. 11.5]). Let X be a manifold equipped with a hermitian metric, and let $Z \subset X$ be a totally geodesic submanifold for which $H^{0}\left(Z, \Theta_{Z}\right)=0$. Then the tangent bundle sequence for $Z \subset X$ splits. Hence $H^{0}\left(Z,\left.\Theta_{X}\right|_{Z}\right)=0 \Longleftrightarrow H^{0}\left(Z, N_{Z \mid X}\right)=0$. It follows that $Z$ is rigidly embedded (keeping the target fixed) if and only if the embedding is rigid keeping source and target fixed.

In particular, since $\delta$ is the zero map in this case, it is irrelevant whether $Z$ itself is rigid or not.

\section{Kodaira-Spencer classes and spreading}

The Kodaira-Spencer class of the spread family $f: X \rightarrow S$ from Prop. 1.1 incorporates arithmetic information, since the dual of $T_{o}(S)$ is the complex 
vector space $\Omega_{k / Q} \otimes_{Q}$ C. Also, $\Omega_{\mathscr{X}}^{1} \mid X=\Omega_{X / k}^{1}$, the sheaf of Kähler differentials on the $k$-variety $X$. The dual of the exact sequence (1) then reads

$$
0 \rightarrow \Omega_{X}^{1} \rightarrow \Omega_{X / k}^{1} \rightarrow \Omega_{k / Q} \otimes_{C} \Theta_{X} \rightarrow 0 .
$$

The extension class of the dual of the above sequence is the KodairaSpencer class for the spread family $f: X \rightarrow S$ at $o$. It depends on the choice of the field $k$ :

$$
\kappa_{X / k} \in H^{1}\left(\operatorname{Hom}_{\circlearrowleft_{X}}\left(\Omega_{X}^{1}, \Omega_{k / \mathbf{Q}} \otimes_{\mathbf{C}} \Theta_{X}\right)\right) \simeq \operatorname{Hom}_{\mathbf{C}}\left(T_{o} S, H^{1}\left(X, \Theta_{X}\right)\right) .
$$

Corollary 1.5. The spread family from Prop. 1.1 is regular. It is a trivial deformation if and only if the Kodaira-Spencer class (3) vanishes.

Proof. To see regularity, first observe that $\operatorname{dim} H^{1}\left(X, \Theta_{X}\right)$ depends on the isomorphism class of $X$ as an abstract algebraic variety. Secondly, since all $X_{s}, s \in S$ with the property that $s$ corresponds to a transcendental number are mutually isomorphic as abstract algebraic varieties, $\operatorname{dim} H^{1}\left(X_{s}, \Theta_{X_{s}}\right)$ is the same for all such $s \in S$. This set corresponds to points in $S$ not lying on any proper subvariety of $S$ and hence is dense in $S$. Upper semicontinuity of $\operatorname{dim} H^{1}\left(X_{s}, \Theta_{X_{s}}\right)$ then implies that this dimension is locally constant, i.e., the family is regular. The result follows from Theorem 1.3 .

\subsection{Rigidity and fields of definition}

Proposition 1.6. 1) Let $X$ be a smooth complex quasi-projective variety. Assume that the Kodaira-Spencer class (3) of some spread family of $X$ vanishes (e.g. in case $X$ is rigid). Then $X$ has a model over a number field, i.e.,

$$
X \simeq X^{\prime} \otimes_{\overline{\mathbf{Q}}} \mathbf{C}, \quad X^{\prime} \text { is defined over } \overline{\mathbf{Q}},
$$

and where the isomorphism is defined over $\mathbf{C}$. This model is unique if $H^{0}\left(\Theta_{X}\right)=$ 0 .

2) Let $(X, Z)$ be a pair of varieties, where $X$ is smooth and $Z \subset X$ a closed embedding. Assume that the Kodaira-Spencer class (2) of a spread family for $(X, Z)$ vanishes (e.g. in case $(X, Z)$ is rigid). Then the pair $(X, Z)$ has a model over a number field, The model is unique if $H^{0}\left(\Theta_{X}(Z)\right)=0$.

3) In the relative situation of a morphism $f: X \rightarrow Y$ between complex quasiprojective varieties suppose that $f$ is rigid. Then $X, Y$ and $f$ have a model over 
$\overline{\mathrm{Q}}$.

4) In the relative situation, suppose that $Y$ is defined over $\overline{\mathbf{Q}}$ and that $f$ is rigid fixing the target. Then the same conclusion as in 3 ) holds.

Proof. 1) Rigidity implies that the fibers of any sufficiently small deformation of $X$ are isomorphic to $X$. This holds in particular for the spread $f: X \rightarrow S$ from Prop. 1.1. So, if $s \in S(\overline{\mathbf{Q}})$, one has an isomorphism $X_{s} \simeq$ $X_{o}=X$ and since $X_{S}$ is defined over $\overline{\mathbf{Q}}, X$ has a model over $\overline{\mathbf{Q}}$. If, moreover, $H^{0}\left(\Theta_{X}\right)=0$ there is no non-trivial deformation of $\mathrm{id}_{X}$ and the isomorphism $X_{s} \simeq X_{o}$ is unique (compare with the definition above).

2) The argument is as for 1), using an obvious variant of Prop. 1.1. for pairs. See remark 1.2.

Note that 3) and 4) can be reduced to embeddings, since $f$ is rigid if and only the embedding of graph of $f$ in $X \times Y$ is a rigid morphism, and the graph is defined over $\overline{\mathbf{Q}}$ precisely when $f$ is. For embeddings $i: X \hookrightarrow Y$, to find a variety over which to spread, start with equations for $Y$ and let $k_{1}$ be the field extension of $\mathbf{Q}$ obtained by adjoining the coefficients. The embedding is then specified by supplementary equations whose coefficients are adjoined to $k_{1}$. The resulting field $k=\overline{\mathbf{Q}}(S)$ is the function field of the base variety $S$. Observe that if the variety $Y$ is defined over a number field, $k_{1}$ is also a number field and then $S$ parametrizes a deformation of $X$ in the fixed variety $Y$. Rigidity in both cases ensures that the embedding has a model over a number field.

Examples 1.7. 1. Fake projective planes are compact complex surfaces of general type with $p_{g}=q=0$ and with $K^{2}=9$. They are known to be quotients of the complex unit 2-ball by an arithmetic subgroup, and are also known to be rigid. See [Pr-Y, $\mathrm{Pr}-\mathrm{Y}-2]$.

2. Let $S$ be a Beauville surface [Be, Exercise X.13.(4)] and [Ba-C-G]. These are certain minimal surfaces of general type with $K^{2}=8, p_{g}=q=0$. Such a surface is rigid [Cat] and so, by Proposition 1.6, it has a model over $\overline{\mathbf{Q}}$. Its complex conjugate cousin, also a Beauville surface, is rigid as well.

$\mathrm{By}[\mathrm{Ba}-\mathrm{C}-\mathrm{G}]$, there are a two more types of surfaces similar to Beauville's examples in that they are all quotients of a product of two curves of genera $>1$ by a freely acting finite group $G$ and having moduli spaces of dimension 0 . Here $G$ is one of two non-abelian groups of order 256. The first gives an example whose moduli space consists of three 0-dimensional components, the second group leads to a unique example. 
The next result gives an application in the relative setting. It leads up to Belyǐ curves:

Proposition 1.8. Suppose $X, Y$ are smooth projective of the same dimension, $p: X \rightarrow Y$ is a surjective finite morphism with smooth branch locus $B \subset Y$. Assume that $Y$ is rigid and that $B$ is rigidly embedded in $Y$. Then $X$ has a model over a number field.

Proof. One constructs a spread of the morphism $p: X \rightarrow Y$ as in the proof of Cor. 1.6. 3. Call it $\tilde{p}: X \rightarrow Y \times S$. We do not now that $p$ is rigid. But the induced deformation of $f$, the family $X \rightarrow Y \times S \rightarrow S$, is differentiably locally trivial over $S$ and so the topological structure of the fibers $p_{s}$ of the map $\tilde{p}$ does not vary. Away from the branch locus, the map $p_{s}$ is a finite étale cover and so the complex structure on

$$
X_{s}^{0}:=p_{s}^{-1}\left(Y-B_{s}\right) \subset X_{s}
$$

is locally determined by the complex structure on $Y-B_{s}$, which by rigidity of the embedding of $B$ in $Y$ is independent of $s$. The manifold structure of $X_{s}$ is fixed and so it only has to be checked that the complex structure on it is completely determined by the complex structure on the Zariski open subset $X_{s}^{0}$.

To show this, note that holomorphic functions on $Y$ are bounded near the branch locus and so, by Riemann's extension theorem, their lifts to $X_{s}^{0}$ can be extended uniquely to $X_{s}$. So indeed, up to isomorphism, the complex structure on $X_{s}$ does not depend on $s$. As before, pick any $s \in S$ defined over $\overline{\mathbf{Q}}$ (which exists since $S$ is by construction defined over $\overline{\mathbf{Q}}$ ). Then, not only $Y_{S}$ is defined over $\overline{\mathbf{Q}}$, but also $X_{S}$ is, and hence, by rigidity, so is the variety $X_{o}=X$.

Remark. By [Mu-O, p. 468-473], a variant of the above proof is apparently due to Carlos Simpson.

Examples 1.9. 1. Recall that a Bely̆ curve [Bel] is a complex projective curve admitting a cover to $\mathbf{P}^{1}$ ramified only in the three points $0,1, \infty$. Three distincts points in $\mathbf{P}^{1}$ define a rigid divisor since three distinct points can always be mapped to three given distinct points by a projective transformation of $\mathbf{P}^{1}$. Belyr showed (loc. cit.) that a complex projective curve can be defined over $\overline{\mathbf{Q}}$ if and only if it is isomorphic to a Belyı̆ curve. The above Proposition shows that the fact that Belyi curves are defined over a 
number field is an example of a quite general phenomenon. The converse statement however requires an explicit construction which is very particular to curves. See [Mu-O, Sect. 9.2] for a proof in the style of this paper. 2. For higher dimensional examples, including branched covers of $\mathbf{P}^{2}$ branched in 4 or less lines, see [Pa].

\section{Further examples of models over number fields}

\subsection{Locally symmetric spaces}

Let $D=G(\mathbf{R}) / K$ be a hermitian symmetric domain, $\Gamma$ a torsion free arithmetic subgroup of $G(\mathbf{R})$ and let $X=\Gamma \backslash D$ be the corresponding locally symmetric space. Such $X$ give examples of Shimura varieties for which it is known that they can be defined over a number field. See e.g. [Mi] for background. Shimura varieties will be investigated more in detail below in Section $3 \cdot 3$.

Here I want to present another approach, due to Faltings which is more in the spirit of this note.

Proposition 2.1 ([F]). The pair $(\bar{X}, \partial X)$ has a unique model over $\overline{\mathbf{Q}}$.

Proof. I give a sketch of Faltings' proof.2 The specific Kodaira-Spencer class $\kappa_{(\bar{X}, \partial X)}$ coming from the derivations of $\mathbf{C} / \mathbf{Q}$ given by (3) lands in the vector space $H^{1}\left(\bar{X}, \Theta_{X}(\partial X)\right)$ measuring infinitesimal deformations of the pair $(\bar{X}, \partial X)$. Using harmonic theory, Faltings shows that each of these can be represented by a unique vector valued harmonic form $\mathrm{H} \kappa_{(\bar{X}, \partial X)}$ on $D$ of type $(0,1)$. Moreover, the assigment $(\bar{X}, \partial X) \mapsto \mathrm{H} \kappa_{(\bar{X}, \partial X)}$ is functorial and equivariant with respect to group actions.

Using this property for the various Hecke correspondences, one shows that such a harmonic form is $\Gamma$-invariant for all possible arithmetic subgroups $\Gamma \subset G$. This form lifts to $D$ as a $G(\mathbf{Q})$-invariant harmonic 1 -form with values in the tangent bundle. By density it is then $G(\mathbf{R})$-invariant on $D$. But such a form must vanish. One sees this as follows. By [Helg, Ch. VIII, §7] the complex structure on the tangent space $T_{0} D$ at a any point $o$ of the hermitian symmetric domain $D$ is induced from the action of the

\footnotetext{
${ }^{2}$ For more details and a generalization of the results of $[\mathrm{Cal}-\mathrm{V}]$ to the non-compact situation see [Pe16].
} 
center $Z \simeq \mathbf{U}^{1}$ of the isotropy group of $o$ on $D: z \in Z$ induces multiplication with $z$. Hence, if $\alpha$ is a global $(0,1)$-form on $D$ with values in the tangent bundle point $o$ the action is given by $z^{*}(\alpha)=\left(\bar{z}^{-1} \cdot z\right) \cdot \alpha$. So $Z$-invariance, implies $\alpha(o)=0$. Since $o$ is arbitrary, $\alpha=0$.

Next, one observes that the spread family for the pair $(\bar{X}, \partial X)$ is regular in the Kodaira-Spencer sense. The proof is similar to the proof of Cor. 1.5 . Hence one may apply (a relative variant of) Theorem $\overline{1.3}(\bar{X}, \partial X)$ is rigid, and hence this pair has model over $\overline{\mathbf{Q}}$.

Uniqueness then follows from $H^{0}\left(\bar{X}, \Theta_{\bar{X}}(\partial X)\right)=0$ (no vectorfields can be tangent along the boundary divisor). Faltings gives an explicit argument reducing the proof to the assertion that there exists no $G(\mathbf{R})$-invariant holomorphic vector fields on $D$. For the last assertion in loc. cit. no proof is given, but the argument is similar to what we did before: The element $z \in Z=\{$ center of the isotropy group of $G(\mathbf{R})$ at $o\}$ acts as multiplication with $z$ on tangent vectors at $o$ and so, invariance implies that any global tangent vector field on $D$ invariant under the action of $G(\mathbf{R})$ vanishes at $o$ and hence everywhere.

\subsection{Holomorphic maps into locally symmetric spaces}

As before, let $X=\Gamma \backslash G / K$ be a locally symmetric space of hermitian type. To $D=G / K$ and a parabolic subgroup $P \subset G$ one associates a boundary component $D(P)$ which is also a bounded symmetric domain. Introduce

$$
\text { rank of } D=\ell(D)=\min _{P} \operatorname{dim} D(P) \text {. }
$$

The numbers $\ell(D)$ for $D$ irreducible are collected in Table 1, The rigidity result I use here is due to Sunada:

Proposition 2.2 ([Su] ). With the above notation, let $M$ be projective, $f: M \hookrightarrow$ $X=\Gamma \backslash D$ with $X$ compact, is rigid keeping source and target fixed, whenever $\operatorname{dim} M \geq \ell(D)+1$.

From Prop. 1.4, Cor. 1.6.3, together with the fact that $X$ is defined over $\overline{\mathbf{Q}}$ whenever $\Gamma$ is arithmetic, we deduce:

Corollary 2.3. If moreover, $\Gamma \subset G$ is a neat congruence subgroup, and $M$ is embedded in $D$ as a totally geodesic submanifold, then $M$ has a model over a number field. 
Table 1: Hermitian symmetric domains

\begin{tabular}{|c|c|c|}
\hline Domain & $\operatorname{dim} D$ & $\ell(D)$ \\
\hline$I_{p, q}=\mathrm{SU}(p, q) / \mathrm{S}(\mathrm{U}(p) \times \mathrm{U}(q))$ & $p q$ & $(p-1)(q-1)$ \\
\hline$I I_{g}=\mathrm{SO}^{*}(2 g) / \mathrm{U}(g)$ & $\frac{1}{2} g(g-1)$ & $\frac{1}{2}(g-2)(g-3)$ \\
\hline$I I I_{g}=\mathrm{Sp}(g) / \mathrm{U}(g)$ & $\frac{1}{2} g(g+1)$ & $\frac{1}{2} g(g-1)$ \\
\hline$I V_{n}=\mathrm{SO}^{\circ}(2, n) / \mathrm{SO}(2) \times \mathrm{SO}(n)$ & $n$ & 1 \\
\hline$V=E_{6} / \mathrm{SO}(10) \cdot \mathrm{SO}(2)$ & 16 & 1 \\
\hline$V I=E_{7} / E_{6} \cdot \mathrm{SO}(2)$ & 27 & 8 \\
\hline
\end{tabular}

Examples 2.4. 1. Since the unit ball $B^{n}$ in $C^{n}$ can be represented as the domain $I_{1, n}$ and since $\ell\left(I_{1, n}\right)=0$, all (positive dimensional) geodesically embedded subvarieties of a compact arithmetic quotient of the unit ball have models over a number field.

2. A domain of type $I V_{n}$ with $n \leq 18$ is a parameter space for lattice polarized $K_{3}$ surfaces, and since $\ell\left(I V_{n}\right)=1$, using local Torelli, we deduce that if we have a family of $\mathrm{K}_{3}$ surfaces over a compact base $B$ of dimension $\geq 2$ whose period map is injective and gives a geodesic embdedding, the base manifold $B$ has a model over a number field.

\section{Applications to variations of Hodge structure}

\subsection{Hodge theory revisited}

As a preliminary to the topic of Shimura varieties, it is useful to view a Hodge structure as a representation space for a certain algebraic torus, as observed by Deligne. See e.g. [Del-M-O-S, Chap. I], [Ca-MS-P, Chap. 15].

To explain this briefly, giving a Hodge structure on a real vector space $V$ is the same as giving a morphism

$$
h: \mathbf{S} \rightarrow \mathrm{GL}(V), \quad \mathbf{S}=\underset{\mathbf{C} / \mathbf{R}}{\operatorname{Res}} \mathbf{G}_{m},
$$

where I recall that the Weil restriction $\operatorname{Res}_{\mathbf{C} / \mathbf{R}} \mathbf{G}_{m}$ is just the group $\mathbf{C}^{\times}$considered as a real group. In other words, a real Hodge structure is just a rational (or "algebraic") representation of the torus group $\mathbf{S}$. One sees this by observing that on the complexified vector space $V_{\mathbf{C}}=V \otimes_{\mathbf{R}} \mathbf{C}$ the action 
of $\mathbf{S}$ diagonalizes and the Hodge subspace $V^{p, q} \subset V_{\mathbf{C}}$ by definition is the subspace where $h(z)$ acts as multiplication with $z^{p} \bar{z}^{q}$.

If the Hodge structure has pure weight $k$ this shows up as follows: via the natural inclusion $w: \mathbf{R}^{\times} \rightarrow \mathbf{S}$, the action of $t \in \mathbf{R}^{\times}$is multiplication by $t^{k}$. This motivates introducing

$$
w_{h}=h \circ w: \mathbf{G}_{m} \rightarrow \mathrm{GL}(V) \text {, the weight morphism. }
$$

If, moreover, $V$ has a rational structure, say $V=V_{\mathbf{Q}} \otimes \mathbf{R}$, this weight morphism is obviously defined over $\mathbf{Q}$. When this is the case, one defines the Mumford-Tate group of $h$ as the smallest closed subgroup $M=M(h)$ of $\mathrm{GL}\left(V_{\mathbf{Q}}\right)$ such that $h$ factors through the real algebraic group $M_{\mathbf{R}}$.

Hodge structures coming from geometry carry a polarization, where I recall that a polarization consists of a $\mathbf{Q}$-valued bilinear form $b$ on $V_{\mathbf{Q}}$ satisfying the two Riemann relations

1. $b_{\mathbf{C}}(x, y)=0$ if $x$ is in $V^{p, q}$ and $y$ is in $V^{r, s}$ for $(r, s) \neq(k-p, k-q)$, where $k$ is the weight of the Hodge structure;

2. $\mathrm{i}^{p-q} b(x, \bar{x})>0$ if $x$ is a nonzero vector in $V^{p, q}$.

A Hodge structure is polarizable if such a $b$ exists and then $M$ is known to be reductive. See [Del-M-O-S, Prop. I.3.6], [Ca-MS-P, Prop. 15.2.6]. Using this language, one singles out a CM-Hodge structure as one whose Mumford-Tate group is abelian and hence, by reductivity, an algebraic torus.

Let me next discuss the notion of a variation of Hodge structure. It consists of a local system $\mathbf{V}$ on a smooth quasi-projective variety $S$ of finite dimensional Q-vector spaces, such that all fibers admit a polarizable Hodge structure. More precisely, $\mathbf{V}$ should come from a representation of the fundamental group of $S$ in a finite dimensional vector space $V$ equipped with a non-degenerate bilinear form $b$ such that

1. the locally free sheaf $\mathscr{V}=V \otimes \mathscr{O}_{S}$ carries a descending filtration $\mathscr{F}^{\bullet}$ by holomorphic subbundles;

2. the natural flat connection $\nabla$ on $\mathscr{V}$ lowers degrees of this filtration by at most 1 (Griffiths' transversality);

3. $b$ and $\mathscr{F} \bullet$ induce a polarized Hodge structure in each stalk. 
Given such a variation of Hodge structure, the Hodge structure over $x \in$ $S$ corresponds to $h_{x}: \mathbf{S} \rightarrow \mathrm{GL}(V)$ and its Mumford-Tate group may vary, However, outside a countable union of proper subvarieties, $M=M\left(h_{x}\right)$ is the same, the generic Mumford-Tate group, and a point with this MumfordTate group is called Hodge generic.

The group

$$
G=\operatorname{Aut}(V, b)
$$

is a Q-algebraic group. The representation of $\pi_{1}(S, x)$ in $V$ defining the local system $\mathbf{V}$ preserves the polarization $b$ and the image $\Gamma$ of $\pi_{1}(S, x)$ in $G(\mathbf{R})$ is discrete. It is called the monodromy group of the variation.

Definition 3.1. The connected component of the $\mathbf{Q}-$ Zariski closure of the monodromy group in $G$ is called the algebraic monodromy group.

The group $G(\mathbf{R})$ acts transitively on a so called period domain $D$, which classifies the Hodge structures on $V$ with a fixed set of Hodge numbers polarized by $b$. The obvious map $p: S \rightarrow \Gamma \backslash D$ is holomorphic; it is called the period map. The Griffiths' transversality condition is in general a further constraint. It is vacuous for weight one variations and also for variations of $\mathrm{K}_{3}$-type.

\subsection{Application to variations of weight 1 and 2}

For a weight two variation with Hodge numbers $h^{2,0}=p, h^{1,1}=q$, the period domain has shape $D=\mathrm{SO}(2 p, q) / \mathrm{U}(p) \times \mathrm{SO}(q)$, the $\mathrm{K}_{3}$-case corresponding to $p=1, q=19$. For weight two domains one further introduces the rank $\ell(D)$ of $D$ which generalizes the concept for hermitian symmetric spaces from Table 1 .

$$
\ell(D)= \begin{cases}1 & \text { if } p=1, \\ q-1 & \text { if } p=2, \\ (p-1) t+\epsilon & \text { if } p \geq 3, t=\left\lfloor\frac{1}{2}(q-1)\right\rfloor, \quad \epsilon= \begin{cases}0 & \text { if } q \text { odd } \\ 1 & \text { if } q \text { even. }\end{cases} \end{cases}
$$

One has the following rigidity result:

Theorem 3.2 ([Pe91, Theorem 3.1]). Let $D$ be a period domain for polarized weight 1 or 2 Hodge structures. An immersive period map $f: S \rightarrow \Gamma \backslash D$ with 
$S$ quasi-projective is rigid keeping source and target fixed as soon as $\operatorname{dim} S \geq$ $\ell(D)+1$.

Using Prop. 1.4, as a corollary, we get:

Corollary 3.3. Let $D$ be a period domain for polarized weight 1 or 2 Hodge structure. Let $S$ be quasi-projective and $f: S \rightarrow \Gamma \backslash D$ an immersive period map of rank $\geq \ell(D)+1$. Suppose moreover, that $S$ is geodesically embedded, then $S$ has a model over $\overline{\mathbf{Q}}$.

\subsection{Shimura varieties}

One needs a Hodge theoretic interpretation of Shimura varieties, i.e., varieties the form $X=\Gamma \backslash D$ for which $D=G(\mathbf{R}) / K$ is a Hermitian symmetric domain of non-compact type and $G$ is a connected $\mathbf{Q}$-algebraic group. For details of the discussion that follows see e.g. [Ca-MS-P, Chap. 16,17], [Mi].

A point $x \in D$ turns out to correspond to a unique $h_{x}: \mathbf{S} \rightarrow G_{\mathbf{R}}$ and so a given representation of $G$ in $V$ defines a real Hodge structure. If the representation comes from a $\mathbf{Q}$-representation $\rho: G \rightarrow \mathrm{GL}\left(V_{\mathbf{Q}}\right)$ one might not get a rational Hodge structure. However, we do get a direct sum of such structures (possibly of different weights) if the weight morphism $\rho \circ h_{x} \circ w: \mathbf{R}^{\times} \rightarrow \mathrm{GL}(V)$ is defined over $\mathbf{Q}$. Such representations exist: take the adjoint representation, with $H=\operatorname{Lie} G$ and $\rho=$ ad $: G \rightarrow G L(V)$ : its weight is zero and hence the weight morphism is automatically defined over $\mathbf{Q}$.

The group $G(\mathbf{R})$ acts by conjugation on $h_{x}$. Let $h_{x}^{(g)}$ denote the conjugate of $h_{x}$ by $g \in G(\mathbf{R})$. Then one has the basic equality

$$
h_{g x}=h_{x}^{(g)}
$$

and hence, since $G(\mathbf{R})$ acts transitively on $D$, one may view $D$ as an entire conjugacy class of maps $h: \mathbf{S} \rightarrow G_{\mathbf{R}}$. Each point in $D$ can be identified with such a map since $h=h^{(g)}$ precisely if $g$ belongs to the isotropy group of the corresponding Hodge structure. For clarity, let me write $[h]$ for the point in $D$ corresponding to $h \in \operatorname{Mor}(\mathbf{S}, G(\mathbf{R}))$. Not any $G(\mathbf{R})$-conjugacy class of a morphism $\mathbf{S} \rightarrow G_{\mathbf{R}}$ underlies a Hermitian symmetric domain. For this to be true, such a morphism has to verify certain axioms, as given in [Del]. If this is the case, the corresponding pair $(G, D)$ is called a Shimura datum 
and $D$ is called a Shimura domain. By the previous remarks about the adjoint representation, all Hermitian symmetric domain thus arise with $G$ the group of holomorphic automorphisms of $D$, which is known to be Q-algebraic and of adjoint type.

It makes sense to define the Mumford-Tate group of a point $[h] \in D$ as the smallest closed subgroup $M(h)$ of $G$ such that $h$ factors through the real algebraic group $M(h)_{\mathbf{R}}$. Then $\rho(M(h))$ is the Mumford-Tate group of the Hodge structure $\rho \circ h$. The orbit of $h \in D$ under its Mumford-Tate group $M(h)$ is a holomorphic submanifold of $D$ which turns out to be a Shimura domain for $M(h)$. It is called the submanifold of Hodge type passing through [h]. Its image in $X$ is called a special subvariety.

As recalled above, for a point $[h] \in D$ outside a countable union of proper closed subvarieties in $D$, the Mumford-Tate group is precisely $G$. Call such a point Hodge-generic. For such points, $D$ is the submanifold of Hodge type through $[h]$. At the other end of the spectrum one has the CMpoints in $D$, by definition those points $[h]$ for which $M(h)$ is abelian (i.e. an algebraic torus). In this case it is its own submanifold of Hodge type. Concerning these points, one has:

Lemma 3.4 ([Ca-MS-P, Corr. 17.1.5]). A Shimura subdomain contains a dense set of CM-points.

\subsection{Monodromy and rigidity}

The geometry of the variation is reflected in the algebraic monodromy, which as I recall, is the connected component $M^{\mathrm{mon}}$ of the $\mathbf{Q}$-Zariski closure in $\mathrm{GL}(V)$ of the monodromy group of the variation. Any reductive group such as $M$ has a canonical almost direct product decomposition

$$
M=M^{\text {der }} \cdot(\text { center of } M),
$$

where $M^{\text {der }}$ is the derived subgroup of $M$, its maximal semi-simple subgroup. There are two important results concerning the relation of the two groups:

Theorem 3.5. 1) An, Thm] The algebraic monodromy group is a normal subgroup of the generic Mumford-Tate group. In fact, one has $M^{\text {mon }} \triangleleft M^{\mathrm{der}}$. 2) [An, Prop. 2] If there are CM-points in the variation, this is an equality $M^{\text {mon }}=M^{\text {der }}$. 
Let me now consider a more general situation of a homomorphic map $p: S \rightarrow \Gamma \backslash D$ to a Shimura variety, i.e. $D=G(\mathbf{R}) / K$ is a bounded Hermitian symmetric domain. This defines a polarizable variation of Hodge structures on $S$ where Griffiths' transversality is automatic. Here $\Gamma$ is the monodromy group of the variation. The group that determines the deformations of $p$ is the centralizer of the algebraic monodromy group inside the group $G$ :

$$
G^{\prime}:=Z_{G}\left(M^{\text {mon }}\right) .
$$

Indeed, one has:

Proposition 3.6. Under the assumption that $X=\Gamma \backslash D$ is a Shimura variety, the "period map" $p: S \rightarrow \Gamma \backslash D$ is rigid if and only if $G^{\prime}(\mathbf{R})$ is compact.

Proof. The Lie algebra $\mathfrak{g}$ of $G(\mathbf{R})$ consists of the endomorphisms of $V$ that are skew with respect to $b$. The Cartan involution induces a direct sum decomposition $\mathfrak{g}=\mathfrak{k} \oplus \mathfrak{p}$ where $\mathfrak{k}$ is the Lie algebra of the maximal compact subgroup $K(\mathbf{R}) \subset G(\mathbf{R})$. The Lie algebra has a natural structure of a weight zero Hodge structure inherited from the one on $\operatorname{End}(V)$. Indeed

$$
\mathfrak{g}_{\mathrm{C}}=\mathfrak{g}^{-1,1} \oplus \mathfrak{g}^{0,0} \oplus \mathfrak{g}^{1,-1}, \quad \mathfrak{g}^{0,0}=\mathfrak{k}_{\mathrm{C}} .
$$

The Lie algebra $\mathfrak{g}^{\prime} \subset \mathfrak{g}$ of $G^{\prime}(\mathbf{R})$ consists of those endomorphisms in $\mathfrak{g}$ that commute with the action of the monodromy group. This subalgebra inherits a weight zero Hodge structure and by [Pego, Theorem 3.4], the tangent space to infinitesimal deformations of $p$ is isomorphic to $\left(\mathfrak{g}_{\mathrm{C}}^{\prime}\right)^{-1,1}$ and in this case, as a real space it is isomorphic to $\mathfrak{p} \cap \mathfrak{g}^{\prime}$. Hence $\mathfrak{p} \cap \mathfrak{g}^{\prime}=0$ if and only if $\mathfrak{g}^{\prime}=\mathfrak{k} \cap \mathfrak{g}^{\prime}$ if and only if $G^{\prime}(\mathbf{R})$ is compact.

Observe next that $G^{\prime}$ is also a reductive group of Hermitian type: $D_{2}:=$ $G^{\prime}(\mathbf{R}) / K \cap G^{\prime}(\mathbf{R})$ is a bounded subdomain of $D$ and if $\tilde{S}$ is a universal cover of $S$ with lifting $\tilde{p}: \tilde{S} \rightarrow D$, there is an induced holomorphic map $F$ : $S \times D_{2} \rightarrow D$ extending $\tilde{f}$. This maps parametrizes the deformations of $f$ keeping $S$ and $D$ fixed. If $f$ embeds $\tilde{S}$ as a subdomain $D_{1} \subset D$, i.e. if $f$ is a geodesic embedding, then one has a product situation

$$
\tilde{F}: D_{1} \times D_{2} \hookrightarrow D .
$$

In other words, the deformations of the embedding $\Gamma_{1} \backslash D_{1} \hookrightarrow \Gamma \backslash D$ between two Shimura varieties are parametrized by a Shimura variety of the form $\Gamma_{2} \backslash D_{2}$. By Prop. 1.4 one then concludes: 
Corollary 3.7 ([Ab, §2]). Let $G$ be a $\mathbf{Q}$-algebraic group of Hermitian type, $G_{1} \subset G$ a reductive subgroup, and let $D=G(\mathbf{R}) / K, D_{1}=G_{1}(\mathbf{R}) / K \cap G_{1}(\mathbf{R})$ the corresponding domains. Put $G_{2}=Z_{G} G_{1}, D_{2}=G_{2}(\mathbf{R}) / G_{2}(\mathbf{R}) \cap K$. Let $\Gamma$ be a neat arithmetic subgroup of $G(\mathbf{Q})$ such that $\Gamma_{i}=\Gamma \cap G_{i}(\mathbf{Q}) i=1,2$ is also neat. The embedding $\Gamma_{1} \backslash D_{1} \hookrightarrow \Gamma \backslash D$ between the corresponding Shimura varieties. is rigid with fixed target precisely when $G^{\prime}(\mathbf{R})$ is compact. In particular, the embedding $\Gamma_{1} \backslash D_{1} \times \Gamma_{2} \backslash D_{2} \hookrightarrow \Gamma \backslash D$ is rigid.

Let me next consider the algebraic monodromy group $M^{\text {mon }} \subset G$ from an arithmetic perspective. First recall that for any $\mathbf{Q}$-simple algebraic group $G$ there is a totally real number field $F$ and an absolutely simple $F$-group $\tilde{G}$ such that

$$
G=\underset{F / \mathbf{Q}}{\operatorname{Res}} \tilde{G} .
$$

Here $\operatorname{Res}_{F / Q}$ is the Weil-restriction whereby an $F$-group is viewed in a functorial way as a $\mathbf{Q}$-group. For a real embedding $\sigma: F \hookrightarrow \mathbf{R}$ let $\tilde{G}^{\sigma}$ be the corresponding conjugate of $\tilde{G}$. It is called a factor of $G$. Then

$$
G_{\mathbf{R}}=\prod_{\sigma \in S} \tilde{G}_{\mathbf{R}}^{\sigma}, \quad S \text { the set of embeddings } F \hookrightarrow \mathbf{R} .
$$

Hence, assuming for simplicity that the algebraic monodromy group is simple over $\mathbf{Q}$, one may write:

$$
M^{\text {mon }}=\underset{F / \mathbf{Q}}{\operatorname{Res}} \tilde{M}^{\text {mon }} \Longrightarrow G^{\prime}=\underset{F / \mathbf{Q}}{\operatorname{Res}} Z_{\tilde{G}} \tilde{M}^{\text {mon }} .
$$

In particular, for every factor $\left(\tilde{M}^{\text {mon }}\right)^{\sigma}$ there is a corresponding factor $\tilde{G}^{\prime \sigma}$. This can be used in the weight one case as follows:

Corollary 3.8. Let there be a weight one variation over a quasi-projective variety with $\mathbf{Q}$-simple algebraic monodromy group. Assume that $M^{\text {mon }}$ has no compact factor. Then the variation (and the period map) is rigid.

Proof. In the weight one case, by [Sat80, Prop. IV.4.3], $M^{\mathrm{mon}}(\mathbf{R})$ and $G^{\prime}(\mathbf{R})$ are in a sense "dual": every non-compact factor $\left(\tilde{M}^{\text {mon }}\right)^{\sigma}$ corresponds to a compact factor $\tilde{G}^{\prime \sigma}$. The assumption implies that all factors of $G^{\prime}$ must be compact and so the deformation is rigid.

This result implies a quite curious result that states that non-trivial monodromy at the boundary implies rigidity: 
Proposition 3.9 ([Sa, Th. 8.6]). A weight one variation over a quasi-projective variety $S$ with a non-trivial unipotent element in the monodromy is rigid.

This holds in particular if $S$ is not compact and there is at least one nonfinite local monodromy operator at infinity.

In these instances, if moreover $S$ is geodesically embedded, it has a model over $\overline{\mathbf{Q}}$.

Proof. First I need a result about ranks of simple groups. Recall that a reductive $k$-algebraic group $G$ has $k$-rank zero if it has no $k$-split tori. By $[B 0, \S 6.4]$ this is the case if and only if $G$ has no non-trivial characters over $k$ and no unipotent elements $g \in G(k), g \neq 1$. For $k=\mathbf{R}$, the $\mathbf{R}$-rank is zero precisely when $G_{\mathbf{R}}$ is compact.

Lemma 3.10. If $G$ is a $\mathbf{Q}$-simple group such that $G_{\mathbf{R}}$ has at least one compact factor, then the $\mathbf{Q}-$ rank of $G$ is zero. In particular, $G$ contains no unipotent elements $g \neq 1$.

To show this, as before, write $G=\operatorname{Res}_{F / Q} \tilde{G}$ with $\tilde{G}$ an absolutely simple group defined over a totally real number field $F$.

A character $\chi$ for $G$ induces a character $\chi_{\sigma}$ for $\tilde{G}^{\sigma}$ and any unipotent $g \in G$ gives a unipotent element $g_{\sigma}$ in $\tilde{G}^{\sigma}$. Suppose $\tilde{G}_{\mathbf{R}}^{\sigma}$ is compact. Then $\chi_{\sigma}=1$ and $g_{\sigma}=1$ and also $\chi=1, g=1$. This finishes the proof of the Lemma.

The Lemma implies that the algebraic monodromy group has no compact factors. Hence, by Cor. 3.8 the deformation is rigid.

A similar result can be shown for variations of $\mathrm{K}_{3}$-type:

Proposition 3.11 ([Sa-Zu, Cor. 5.6.3]). Suppose we have a non-isotrivial $\mathrm{K}_{3}$-variation over a quasi-projective variety $S$ with a non-trivial unipotent element in the monodromy. Assume that the variation is not isotrivial. Suppose moreover, that its rank is not 4. Then the variation (and the period map) is rigid, and if $S$ is also geodesically embedded, then $S$ has a model over $\overline{\mathbf{Q}}$.

Proof. Here Lemma 3.10 is used in a different manner.: For a non-isotrivial isotypical variation which is non-rigid, $M^{\mathrm{mon}}(\mathbf{R})$ has one conjugate isomorphic to SL(2, $\mathbf{R})$ with representation space $\mathbf{R}^{2} \otimes \mathbf{R}^{2}$ and the remaining conjugates are $\simeq S U(2)$ with representation space $C^{2}$. It follows from the Lemma that the only possibility to accommodate a non-trivial unipotent element $T$ is when no compact conjugates are present and then the local system has rank 4. 


\subsection{Special subvarieties of Shimura varieties}

Recall (\$3.3) that a special subvariety of a Shimura variety $X=\Gamma \backslash G / K$, or a subvariety of Hodge type, comes from the orbit of a point in $D=G / K$ under its own Mumford-Tate group. In this subsection we study them in more detail.

A morphism of Shimura varieties

$$
X_{1}=\Gamma_{1} \backslash \underbrace{G_{1}(\mathbf{R}) / K_{1}}_{D_{1}} \rightarrow X_{2}=\Gamma_{2} \backslash \underbrace{G_{2}(\mathbf{R}) / K_{2}}_{D_{2}}
$$

is by definition induced by an equivariant morphism of Shimura domains. Such a morphism is given by a morphism $\varphi: G_{1} \rightarrow G_{2}$ of $\mathbf{Q}$-algebraic groups. It then induces a holomorphic maps of Shimura domains $f$ : $D_{1} \rightarrow D_{2}$ by stipulating that $f\left(\left[h_{1}\right]=\left[\left(\varphi \circ h_{1}\right)\right]\right.$ for one hence all points $\left[h_{1}\right] \in D_{1}$. The Mumford-Tate group of $\left[h_{1}\right]$ maps under $\varphi$ to the MumfordTate group of $f\left(\left[h_{1}\right]\right)$. It follows that the subvariety $f\left(D_{1}\right)$ is special in $D_{2}$ : if $\left[h_{1}\right]$ is Hodge generic, then $f\left(\left[h_{1}\right)\right.$ has Mumford-Tate group $\varphi\left(G_{1}\right)$ and $f\left(D_{1}\right)$ is the orbit of this group acting on $f\left(\left[h_{1}\right]\right)$.

Suppose next that $f$ is an embedding. One then may assume that $\varphi$ is also an embedding. It is not hard to see that $f$ is a totally geodesic embedding. See e.g. [Ca-MS-P, Chap 11.5]. Then the conjugate map $f^{\left(g^{\prime}\right)}$, $g^{\prime} \in G_{2}(\mathbf{R})$ is also a totally geodesic embedding. It may or may not arise from a morphism of Shimura domains. Indeed, its image may not have CM-points at all.

The variation of Hodge structure on $D_{2}$ restricts to one on $f^{\left(g^{\prime}\right)} D_{1}$ and this descends to give one on its image in $X_{2}$. The monodromy of this variation is $\Gamma_{1}^{\left(g^{\prime}\right)}$. The connected component $M^{\text {mon }}$ of its Zariski closure in $G_{2}$ acts transitively on $D=f^{\left(g^{\prime}\right)} D_{1} \subset D_{2}$ and so, if it would be the generic Mumford-Tate group $M$ or, which suffices, its derived group, the subdomain $D$ would be of Hodge type. This is the case if $D$ contains a CM-point. Indeed, by Theorem 3.5 one then has $M^{\text {mon }}=M^{\text {der }}$. Concluding, I have shown:

Lemma 3.12. Let $D_{1}, D_{2}$ two Shimura domains and let be $i=f^{\left(g^{\prime}\right)}: D_{1} \hookrightarrow D_{2}$ as above. Then $i$ is a morphism of Shimura domains if and only if $g^{\prime} \in G_{2}(\mathbf{Q})$ if and only if $i\left(D_{1}\right)$ contains a CM-point of $D_{2}$.

This can be used to give another proof of Abdulali's criterion [Ab, Thm. 3.4]: 
Proposition 3.13. Let $i: X_{1} \hookrightarrow X_{2}$ be a totally geodesic embedding of Shimura varieties. If the embedding is rigid, $i\left(X_{1}\right)$ is a special subvariety.

Proof. Since Shimura varieties are defined over a number field (cf. [Mi]), one may apply Cor. 1.6.4. So, if the embedding is rigid, the image is defined over a number field. To show that the image is a special subvariety, by the previous Lemma, it suffices to find a CM-point in the image. But, if $x \in X_{1}$ is a CM-point, then $i(x)$ is also a CM-point since the Mumford-Tate group of the Hodge structure corresponding to $x$ is an algebraic torus and hence, so is the one associated to $i(x)$ since $i$ is defined over $\overline{\mathbf{Q}}$.

Corollary $3 \cdot 7$ then yields examples for weight one Hodge structures:

Examples 3.14. 1. The group $G_{1}=\mathrm{GL}(2)$ can be embedded in $\operatorname{Sp}(g)$ as follows. Set $V_{k}=\left(\mathbf{R}^{2 k}, J_{k}\right), J_{k}=\left(\begin{array}{cc}0 & \mathbf{1}_{k} \\ -\mathbf{1}_{k} & 0\end{array}\right)$. The direct sum $\oplus_{k} V_{1}$ is isomorphic to the symplectic space $V_{k}$. Whence a faithful representation $\rho_{k}$ of 2 :

$$
\left(\begin{array}{ll}
a & b \\
c & d
\end{array}\right) \stackrel{\rho_{k}}{\longrightarrow}\left(\begin{array}{ll}
a \mathbf{1}_{k} & b \mathbf{1}_{k} \\
c \mathbf{1}_{k} & d \mathbf{1}_{k}
\end{array}\right)
$$

For any $k=1, \ldots, g$ the direct sum representation $\rho_{k} \oplus(\operatorname{rank}(g-k)$ trivial representation) induces a holomorphic embedding $\mathfrak{I} \hookrightarrow \mathfrak{I}_{g}$. It gives the non-compact embedded Shimura curves starting from the Shimura datum (SL(2), I I). There is no locally constant factor if and only if $k=g$ and then the embedding is rigid. This follows from Corollary 3.8. These noncompact rigid curves are often called rigid curves of Satake type.

2. There are also examples where $G_{1}$ has compact factors. Here I use again the Satake "duality" mentioned before, but in its precise form as explained in [Sa, §7]. It applies to $G_{1}$ and $G_{1}^{\prime}:=Z_{\mathrm{Sp}(g)} G_{1}$ and gives:

$$
\left(G_{1}\right)_{\mathbf{R}} \simeq \mathrm{SL}(2) \times \underbrace{\mathrm{SU}(2) \times \cdots \times \mathrm{SU}(2)}_{m-1} \Longrightarrow\left(G_{1}^{\prime}\right)_{\mathbf{R}} \simeq \mathrm{SO}(2) \times \underbrace{\mathrm{SU}(1) \times \cdots \times \mathrm{SU}(1)}_{m-1}
$$

The latter group is compact and hence the deformation is rigid. There are indeed examples of such embeddings, the Mumford type curves. See [Ad, $\$ 6.2]$. 


\section{Applications to Higgs bundles}

\subsection{Basic notions}

A Higgs bundle 3 over a complex manifold $B$ is a pair $(\mathscr{V}, \tau)$ of a holomorphic bundle together with an $\operatorname{End}(\mathscr{V})$-valued 1-form $\tau$ such that $\tau \circ \tau=0$. The form $\tau$ can also be viewed as a Higgs field, a homomorphism $\tau: \mathscr{V} \rightarrow$ $\mathscr{V} \otimes \Omega_{B}^{1}$. A graded Higgs bundle is a Higgs bundle such that $\mathscr{V}=\oplus_{r} \mathscr{V}^{r}$, with $\mathscr{V}^{r}$ locally free and such that $\tau \mid \mathscr{V}^{r}: \mathscr{V}^{r} \rightarrow \mathscr{V}^{r-1} \otimes \Omega_{B}^{1}$.

The standard example comes from polarized complex variations of Hodge structures on $B$. Recall [Simp, §4] that such a system consist of

- a local system of $\mathbf{C}$-vector spaces $\mathbf{V}$ equipped with a flat non-degenerate bilinear form. In other words, if $\pi$ is the fundamental group of $B$ based at $o \in S, \mathbf{V}$ comes from a representation $\rho: \pi \rightarrow \mathrm{O}(V, b), V$ the fiber of $\mathbf{V}$ at $o$;

- a direct sum decomposition $\mathbf{V} \otimes C_{B}^{\infty}=\oplus_{r} \mathscr{T}_{\infty}^{r}$ into locally free $C_{B}^{\infty}-$ modules such that

- the hermitian form $h(x, y)=(-1)^{r} b(x, \bar{y})$ on $\mathscr{V}_{\infty}^{r}$ is positive definite and the above decomposition is $h$-orthogonal;

- the natural flat connection $\nabla$ on $\mathbf{V} \otimes C_{B}^{\infty}$ obeys

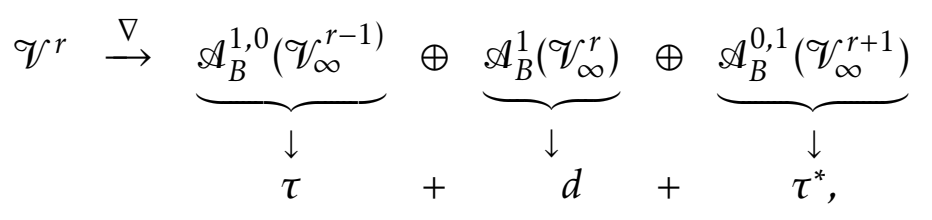

where $\tau^{*}$ is the $h$-adjoint of $\tau$.

These demands imply that $\mathscr{F}^{p}=\oplus_{r \geq p} \mathscr{T}_{\infty}^{r}$ is a holomorphic subbundle of $\mathbf{V} \otimes \mathscr{O}_{B}$ and that Griffiths' transversality holds. This filtration is the Hodge filtration. It also follows that the holomorphic bundle

$$
\mathscr{V}=\oplus_{p} \mathscr{F}^{p} / \mathscr{F}^{p+1}, \quad \mathscr{C}_{B}^{\infty}\left(\mathscr{F}^{p} / \mathscr{F}^{p+1}\right)=\mathscr{V}_{\infty}^{p}
$$

with the underlying local system $\mathbf{V}$ admits the structure of a graded Higgs bundle with $\tau$ the Higgs field. Flatness (i.e., $\nabla \circ \nabla=0$ ) implies the Higgs

\footnotetext{
${ }^{3}$ For more details on Higgs bundles see e.g. [Ca-MS-P, Chapter 13].
} 
condition $\tau \circ \tau=0$. Moreover, the Chern connection, that is, the unique holomorphic connection on this Higgs bundle which is metric with respect to the hermitian metric $h$ turns out to be $\bar{\partial}+\tau$. So on any subbundle on which $\tau=\tau^{*}=0$, the flat connection $\nabla$ induces the Chern connection and so the metric $h$ coincides with the flat metric. Moreover, such a subbundle comes from a local subsystem of $\mathbf{V}$ since it is preserved by $\nabla$. Also, it is unitary since it admits the flat unitary metric $h$. This holds in particular for the largest subbundle for which $\tau=\tau^{*}=0$ :

$$
\mathcal{U}=\mathbf{U} \otimes \mathscr{O}_{B} \text { : the maximal unitary Higgs subbundle. }
$$

There is an $h$-orthogonal splitting

$$
\mathbf{V}=\mathbf{U} \oplus \mathbf{W}, \quad \mathbf{W}=\mathbf{U}^{\perp} .
$$

\subsection{Logarithmic variant}

If $B$ is quasi-projective, one usually considers Higgs bundle with logarithmic growth near the boundary. To explain this, assume for simplicity that $\operatorname{dim} B=1$ and that $B$ get compactified to a a smooth projective curve $\bar{B}$. Then the boundary $\Sigma=\bar{B}-B$ consists of finitely many points. A graded logarithmic Higgs bundle $\mathscr{V}=\oplus_{p} \mathscr{V}^{p}$ on $B$, with $\mathscr{V}^{p}$ locally free, by definition admits a Higgs field with components

$$
\tau: \mathscr{V}^{p} \rightarrow \mathscr{V}^{p-1} \otimes \Omega \frac{1}{\bar{B}}(\log \Sigma) .
$$

For a variation of Hodge structure on $B$ with unipotent monodromy at the punctures, one lets $\mathscr{V}$ be the associated graded of the Deligne extended Hodge filtration. Then the Gauss-Manin connection induces a Higgs field as above.

Even more is true. Choose a coordinate patch $(\Delta, t)$ around a puncture and let $T$ be the (unipotent) local monodromy operator around the puncture. For $v$ a local holomorphic section of $\mathscr{V}$ on the disc, write

$$
\nabla v=R \frac{d t}{t}, \quad R \in \operatorname{End}\left(\left.\mathscr{V}\right|_{\Delta}\right) .
$$

Then

$$
N:=R(0)=\log (T) \in \operatorname{End}(V)
$$


and the Higgs field at the puncture is given by

$$
\tau(0): \mathscr{V}_{0} \rightarrow \mathscr{V}_{0} \otimes \frac{d t}{t}, \quad v^{p} \mapsto\left(\mathrm{Gr}^{p} N\right) v^{p} \otimes \frac{d t}{t}
$$

Suppose $k$ is the first index in the grading for which $\mathscr{V}^{k} \neq 0$ and $k+w+1$ the last. Then the number $w$ is called the width.

In this general setting, one says that for a Higgs bundle of width $w$, the Higgs field is generically maximal if for all $p \in[k, k+w+1]$ one has $\mathscr{V}^{p} \neq 0$ and if, moreover, $\tau \mid \mathcal{V}^{p}$ generically an is an isomorphism for $p=k, \ldots, p+w$.

\subsection{Rigid maximal Higgs subsytems}

The following rigidity result [Vie-Z, Lemma 3.1], stated without proof, can be formulated in a slightly different way which fits better within the general framework set up so far:

Proposition 4.1. Let $B$ be a smooth quasi-projective variety, $\mathbf{V}$ be a local system on $B$ of finite dimensional $\mathbf{Q}$-vector spaces and let $\mathbf{W}_{\mathbf{C}}$ a subsystem of $\mathbf{V} \otimes \mathbf{C}$. Suppose $\mathbf{W}_{\mathbf{C}}$ is rigid as a subsystem of $\mathbf{V} \otimes \mathbf{C}$. Then $\mathbf{W}_{\mathbf{C}}$ is defined over $\overline{\mathbf{Q}}$ in the sense that $\mathbf{W}_{\mathbf{C}}=\mathbf{W} \otimes \mathbf{C}$, where $\mathbf{W}$ is a local system of $F$-vector spaces for some number field $F$.

Proof. Let $\pi$ be the fundamental group of $B(\mathbf{C})$ based at $o \in B(\mathbf{C})$ and let $V$ be the fiber at $o$ of $\mathbf{V}_{\mathbf{C}}$, considered as a $\pi$-representation space. The group $\pi$ acts on the Grassmannian $G(r, V)$ of $r$-dimensional subspaces $W \subset V$, where $r=\operatorname{rank} \mathbf{W}_{\mathbf{C}}$. A fixed point $[W]$ of this action corresponds to a complex subsystem of $\mathbf{V} \otimes \mathbf{C}$. More precisely, the corresponding $\pi$-invariant subspace is the fiber $U_{[W]}$ at $[W]$ of the tautological bundle $U \rightarrow G(r, V)$. The spread of the point $[W]$ is a subvariety $Y \subset G(r, V)$ contained in the locus of fixed points of the $\pi$-action, because this action is defined over Q. The tautological subbundle over $Y$ gives a deformation of $\mathbf{W}_{\mathbf{C}} \subset \mathbf{V}_{\mathbf{C}}$, and so rigidity implies that $Y(\overline{\mathbf{Q}})=[W]$, an isolated point. Hence the local system $\mathbf{W}_{\mathbf{C}}$, which corresponds to $U_{[W]}$, is defined over $\overline{\mathbf{Q}}$.

As a direct application, one has:

Proposition 4.2 ([Vie-Z, Lemma 3.3] ). Let $B=\bar{B}-\sum$ as above. Suppose that $\mathbf{V}$ underlies a polarizable $\mathbf{Q}$-variation of Hodge structure and let $(\mathscr{V}, \sigma)$ the corresponding graded logarithmic Higgsbundle over B with unipotent monodromy 
around points of $\Sigma$. Let $\mathbf{V}_{\mathbf{C}}=\mathbf{U} \oplus \mathbf{W}$ the splitting (4). Suppose that the logarithmic Higgs subbundle $\mathscr{W}$ corresponding to $\mathbf{W}$ is a generically maximal Higgs subbundle. Then the splitting is defined over $\overline{\mathbf{Q}}$.

Proof. To show how this result is implied by Proposition 4.1, it is enough to show that $\mathbf{W}$ is rigidly embedded in $\mathbf{V}$. Again, with $V$ a typical fiber of $\mathbf{V}$, small deformations of $\mathbf{W}$ are parametrized by the tangent space to the fixed locus under the $\pi$-action on the Grassmannian $G(r, V)$ at a $\pi$ invariant point $[W]$. A tangent vector is therefore represented by a homomorphism of local systems

$$
q: \mathbf{W} \rightarrow \mathbf{V} / \mathbf{W}=\mathbf{U}
$$

which is compatible with the structure as a complex system of Hodge bundles: a small deformation of $\mathbf{W}$ within $\mathbf{V}$ inherits this structure from the one on $\mathbf{V}$ and the map $q$ is the embedding of the deformed $\mathbf{V}$ followed by restriction to $\mathbf{U}$. But the Higgs field for the left hand is generically an isomorphism while on the right hand it is zero. This is impossible unless $q=0$.

Remark. A variant of this (loc. cit.) is when $\mathbf{W}$ is a direct sum of complex systems of Hodge bundles of different widths, all with generically maximal Higgs field. Then almost the same argument shows that also this splitting is defined over $\overline{\mathbf{Q}}$. There is one subtlety here: one has to compare projections between complex systems of different widths and then one needs semi-simplicity for variations of Hodge structures. This property is a highly non-trivial consequence of another rigidity property due to Schmid [Sch]. See [Pe-St, §5] for details.

One can say more, if there are punctures with infinite local monodromy: Proposition 4.3. The situation is as in Prop. 4.2. In particular, all local monodromy operators at the boundary are unipotent. Assume that at least one local monodromy operator has infinite order. Then the splitting $\mathbf{V}=\mathbf{U} \oplus \mathbf{W}$ from (4) is defined over $\mathbf{Q}$ and $\mathbf{U}$ extends as a local system to $\bar{B}$. The monodromy of this last system is finite.

Proof. The property that a Higgs field is an isomorphism on $B$ extends to $\bar{B}$. If all graded fields $\tau_{p}$ are isomorphisms, at a puncture (5) implies that the $\mathrm{Gr}^{p} N$ are isomorphisms and hence that $N$ is an isomorphism. This holds for $\mathscr{W}$, while the fact that the Higgs field for $U$ remains zero at a 
puncture implies that the local monodromy for $\mathbf{U}$ is the identity and thus that this local system extends to $\bar{B}$.

Suppose that we have a splitting as above, valid over a Galois extension $F / Q$. The property that $N$ is an isomorphism or zero is preserved by the action of the Galois group $\mathscr{G}$. It follows that for $\sigma \in \mathscr{G}$ the natural inclusion followed by projection

$$
\mathbf{U}^{\sigma} \rightarrow \mathbf{V} \rightarrow \mathbf{V} / \mathbf{U}=\mathbf{W}
$$

sends the fiber $\mathbf{U}_{s}^{\sigma}$ at a puncture $s \in \sum$ to zero. In other words $\mathbf{U}_{s}=\mathbf{U}_{s}^{\sigma}$ for all $\sigma \in \mathscr{G}$ and so this fiber is defined over $\mathbf{Q}$. Since $\mathbf{U}$ extends to $\bar{B}$, and since the entire monodromy action is defined over $\mathbf{Q}$, the local system $\mathbf{U}$ which is built from the monodromy representation on some fiber $\mathbf{U}_{s}$ is then defined over $\mathbf{Q}$. The polarization is defined over $\mathbf{Q}$ as well and hence $\mathbf{W}=\mathbf{U}^{\perp}$ is defined over $\mathbf{Q}$. The finiteness of the monodromy follows since the system is defined over $\mathbf{Q}$ and the polarization $h$ on it is a positive definite $\mathbf{Q}$-valued form preserved by the monodromy.

Example 4.4 (An interesting Shimura curve in the Torelli locus). The above result definitely fails when $B=\bar{B}$ : the global monodromy of $\mathbf{U}$ may be infinite. The simplest example from [M-O, Example 5.1] is a Shimura curve and can be described as follows. Consider the family of projective curves with affine equation

$$
y^{5}=x(x-1)(x-t) .
$$

This gives a family $C_{t}$ over $\mathbf{P}^{1}$ of genus 4 curves. The fibers are smooth for $t \neq 0,1, \infty$. Note however that local monodromy operators are quasiunipotent in this case, but this does not really matter since this could be taken care of by a finite branched cover of $\mathbf{P}^{1}$. For simplicity this will not be done since the above analysis still works after some minor modification.

Let $\zeta_{5}$ be primitive root of unity. Then the cyclic group $\mathbf{Z} / 5 \mathbf{Z}$ generated by $(x, y) \mapsto\left(\zeta_{5} y, x\right)$ preserves $C_{t}$ and the Hodge structure $H^{1}\left(C_{t}\right)$ of weight one admit an action of $\mathbf{Z} / 5 \mathbf{Z}$. Let $F=\mathbf{Q}\left(\zeta_{5}\right)$. The Galois group $\mathscr{G}$ of $F / Q$ is generated by the element $\sigma$ which sends $\zeta_{5}$ to $\zeta_{5}^{2}$. It permutes the eigenspaces of $\mathbf{Z} / 5 \mathbf{Z}$ acting on $V_{t}=H^{1}\left(C_{t}, \mathbf{C}\right)$ as in the following table. Next, consider the splitting of the corresponding Higgs bundle. The Higgs bundle splits also in eigenspace subbundles; the Higgs field is zero for the subbundles corresponding to the first two rows and an isomorphism for 
Table 2: Eigenspaces for $\mathbf{Z} / 5 \mathbf{Z}$ on $V_{t}$

\begin{tabular}{|c|c|c|}
\hline Eigenvalue & $h^{1,0}$ & $h^{0,1}$ \\
\hline$\zeta_{5}$ & 0 & 2 \\
\hline$\sigma\left(\zeta_{5}\right)=\zeta_{5}^{2}$ & 2 & 0 \\
\hline$\sigma^{2}\left(\zeta_{5}\right)=\zeta_{5}^{4}$ & 1 & 1 \\
\hline$\sigma^{3}\left(\zeta_{5}\right)=\zeta_{5}^{3}$ & 1 & 1 \\
\hline
\end{tabular}

those corresponding to the last two. So the Higgs bundle is maximal and one has

$$
\mathbf{U}=V_{\zeta_{5}} \oplus V_{\zeta_{5}^{2}}, \quad \mathbf{V}=V_{\zeta_{5}^{4}} \oplus V_{\zeta_{5}^{3}} .
$$

If $M^{\text {mon }}$ is the algebraic monodromy group of this family, one has a corresponding splitting

$$
M^{\mathrm{mon}}(\mathbf{R})=\mathrm{SU}(2) \times \mathrm{SU}(1,1) .
$$

The actual monodromy group $\Gamma$ in this case is dense in both factors and hence cannot be finite for the local system U. Proposition 4.3 then implies that the local monodromy around the punctures cannot be of infinite order. Indeed, one can show that the local monodromy operators are all of order 5 in this case. Hence the period map extends over the punctures and the period map embeds the base curve as a compact curve in the period domain. This is consistent with the fact that fibers over the punctures are of so called compact type: their generalized Jacobians are products of principally polarized Abelian varieties whose dimensions sum up to 4 .

Note also that this is an elementary example giving a negative answer to the following question of Fujita [Ue]:

"for a family $f: X \rightarrow B$ of complex algebraic manifolds over a curve $B$, is the sheaf $f_{*} \omega_{X / B}$ is semi-ample?"

In the above example the latter sheaf is just the graded part $\mathscr{H}^{1,0}=$ $\mathcal{U}^{1,0} \oplus \mathcal{V}^{1,0}$ of the Higgs bundle and while the second bundle is ample, the first is flat and would be semi-ample if and only if its global monodromy would be finite, but this group is dense in $M^{\mathrm{mon}}(\mathbf{R})$. Compare also the much more elaborate examples in [Cat-D]. 


\section{References}

[Ab] Abdulali, S: Conjugates of strongly equivariant maps, Pac. J. Math. 165 207-216 (1994)

[Ad] Addington, S.: Equivariant holomorphic maps of symmetric domains, Duke Math. Journal 55 (1987), 65-88

[An] ANDRÉ, Y.: Mumford-Tate groups of mixed Hodge structures and the theorem of the fixed part. Compositio Math. 82 (1992) 1-24

[Ba-C-G] Bauer, I., F. Catanese and F. Grunewald: Surfaces with $p_{g}=q=0$ isogenous to a product. ArXiv : math /0610267

[Be] Beauville, A.: Complex algebraic surfaces, Cambridge Univ. Press (1983) (translation of Surfaces algébriques complexes, Astérisque 54 Soc. Math. France, Paris (1978)).

[Bel] BeLy̌̆, G. V.: Galois extensions of a maximal cyclotomic field. (Russian) Izv. Akad. Nauk SSSR Ser. Mat. 43 (1979), $267-276$

[Bo] Borel, A.: Linear algebraic groups, in Proc. Symp. Pure Math. 9 (1966) 3-19

[Cal-V] Calabi, E. and E. Vesentini: On compact, locally symmetric Kähler manifolds, Ann. of Math. 71 (1960) 472-507.

[Ca-MS-P] Carlson, J., S. Müller-Stach and C. Peters: Period maps and period domains, Second edition, C.U.P., Cambridge (2017).

[Cat] Catanese, F.: Fibred Surfaces, Varieties Isogenous to a Product and Related Moduli Spaces, American Journal of Mathematics, 122 (2000), 1-44

[Cat-D] Catanese, F. and M. Dettweiler: Answer to a question by Fujita on Variation of Hodge Structures, arXiv: 1311.3232 [math.AG] 
[Del] Deligne, P.: Variétés de Shimura: interprétation modulaire, et techniques de construction de modèles canoniques in Automorphic forms, representations and L-functions (Proc. Sympos. Pure Math., Oregon State Univ., Corvallis, Ore., 1977), Part 2 247-289, Proc. Sympos. Pure Math., XXXIII, Amer. Math. Soc., Providence, R.I., (1979)

[Del-M-O-S] Deligne, P., J. S. Mirne, A. Ogus and K. Shin: Hodge cycles, motives and Shimura varieties, Lecture Notes in Mathematics, Springer Verlag, Berlin, etc. 900, (1982)

[F] Faltings, G.: Arithmetic varieties and rigidity. in Seminar on number theory, (Paris, 1982/1983), Progr. Math., 51, Birkhäuser Boston, Boston, MA, (1984) 63-77

[G-G] Green, M. and P. Griffiths: On the tangent space to the space of algebraic cycles on a smooth algebraic variety, Annals of Math. Studies 157 Princeton Univ. Press (2005)

[Helg] Helgason, S.: Differential Geometry, Lie Groups and Symmetric Spaces, Academic Press, New York/London (1978)

[K-S] Kodaira, K. and D. Spencer: On deformations of complex structure I,II, Ann. Math. 67 (1957) 328-466.

[Mi] Milne, J.: Introduction to Shimura varieties, available online as http: / /www. jmilne.org/math/xnotes/svi.pdf (2004)

[M-O] Moonen, B. And F. Oort: The Torelli locus and special subvarieties, Handbook of moduli. Vol. II, 549-594, Adv. Lect. Math. (ALM), 25, Int. Press, Somerville, MA, 2013.

[Mu Mumford, M.: Hirzebruch's proportionality theorem in the non-compact case. Invent. Math. 42 239-272 (1977)

[Mu-O] Mumford, M. and T. Oda: Algebraic Geometry II, Text and readings in mathematics 73 , Hindustan book agency (India) (2015)

[Pa] Paranjape, K. H. : A geometric characterization of arithmetic varieties. Proc. Indian Acad. Sci. Math. Sci. 112 (2002) 383-391. 
[Pego] Peters, C.: Rigidity for variations of Hodge structure and Arakelov-type finiteness theorems, Comp. Math. 75 (1990) 113-126.

[Pe91] Peters, C.: On the rank of non-rigid period maps in the weight one and two case, in Complex algebraic varieties, Proceedings, Bayreuth 1990 157-165, Lect. Notes in Math. 1507 Springer-Verla, Berlin etc. (1991)

[Pe16] Peters, C.: On rigidity of locally symmetric spaces. Preprint 2016.

[Pe-St] Peters, C. and J. Steendrink: Monodromy of variations of Hodge structure, Acta Appl. Math. 75 183-194 (2003)

[Pr-Y] Prasad, G. and S. K. Yeung: Fake projective planes, Invent Math 168, 321-370 (2007), arXiv:math// 0512115

[Pr-Y-2] Prasad, G. and S.K. Yeung: Addendum to 'Fake Projective Planes', http: / /arXiv.org/abs/ogo6. 4932

[Sa] SAIto, M.-H.: Classification of non-rigid families of abelian varieties, Tohoku Math. J. 45 (1993) 159-189.

[Sa-Zu] Saito, M.-H. ANd S. Zucker: Classification of nonrigid families of $\mathrm{K}_{3}$-surfaces and a finiteness theorem of Arakelov type, Math. Ann. 289 (1991) 1-31.

[Sat67] SATAKe, I.: Symplectic representations of algebraic groups satisfying a certain analyticity condition, Acta Math. 117 (1967) $215^{-279}$

[Sat8o] Satake, I.: Algebraic Structures of Symmetric Domains, Publ. Math. Soc. Japan 14, Iwanami Shoten, Japan, and Princeton UN. Press (1980)

[Sch] Schmid, W.: Variation of Hodge structure: the singularities of the period mapping, Invent. Math. 22, 211-319 (1973)

[Sern] Sernesi, E.: Deformations of Algebraic Schemes, SpringerVerlag Berlin, Heidelberg (2006) 
[Simp] Simpson, C.: Higgs bundles and local systems, Publ. Math. IHES 75 (1992) 5-95

[Sh] Shimura, G.: Algebraic varieties without deformations and Chow variety, J. Math. Soc. Japan 20 (1968) 336-341

[Su] SunAda, T.: Holomorphic mappings into a compact quotient of symmetric bounded domain. Nagoya Math. J. 64 (1976), 159-175.

[Ue] Ueno, K. (editor): Open problems: Classification of algebraic and analytic manifolds, in Classification of algebraic and analytic manifolds, Proc. Symp. Katata/Jap. 1982, Progress in Mathematics, 39, Birkhäuser, Boston, Mass. (1983), 591-630.

[Vie-Z] VIEHWeg, E. AND K. Zuo: A characterization of certain Shimura curves in the moduli stack of abelian varieties. J. Differential Geom. 66 233-287 (2004). 